\title{
Analysis of diagnosis and treatment of heterotopic pancreas
}

\author{
Shipeng Song ${ }^{*}$ Rui Liu, Shengbin Zhang, Tianyong Zhao, Jin Zhao, Zhijian Zhang \\ The Third Affiliated Hospital of Inner Mongolia Medical University, Hohhot, China
}

Received: January 1, 2015

DOI: $10.14725 /$ dcc.v2n2p23

\author{
Accepted: February 2, $2015 \quad$ Online Published: June 15, 2015 \\ URL: http://dx.doi.org/10.14725/dcc.v2n2p23
}

\begin{abstract}
A case of heterotopic pancreas in the Third Affiliated Hospital of Inner Mongolia Medical University was recorded and analyzed on the basis of diagnosis, physical examination and treatment. Misdiagnosis of gastrointestinal stromal tumor (GIST) is very common since it is a rare disease. So this paper aims to enhance the doctors' awareness of GIST during clinical practice.
\end{abstract}

Key Words: Heterotopic pancreas, Diagnosis, Treatment

\section{Medical record}

\subsection{General information}

A 35-year-old man was admitted to our hospital on November 22, 2011 due to middle and upper abdominal pain and melena of three months duration. He suffered from intermittent epigastric pain, especially after starvation, three months prior to his admission, accompanied by nausea, heartburn, acid reflux. He passed black stool, an average of one or two times per day about $50 \mathrm{ml}$. There were no signs of vomiting, fever, chills, cutaneous or sclera icterus. The patient had oral administration of omeprazole capsules $(20 \mathrm{mg}$, once a day), sucralfate tablets ( $1 \mathrm{~g}$, three times a day), colloidal bismuth pectin capsules $(150 \mathrm{mg}$, three times a day), without any clinical effect. He was then referred to the gastroenterology clinic of our hospital for duodenal digestive endoscopic ultrasound examination, indicating the appearance of mass in the muscle layer of duodenum. He was low in spirit, poor appetite and with weight loss around $2.5 \mathrm{~kg}$ since the onset of the symptoms. His previous personal, family and vaccination history were noncontributory.

\subsection{Physical examination}

T: $36.2{ }^{\circ} \mathrm{C}, \mathrm{P}: 80$ beats/min, R: 20 times/min, BP: 110/75 $\mathrm{mmHg}$. No cutaneous or sclera icterus was included. Superficial lymph nodes were not found to be enlarged. Double lung breath sounds resonance. Flat abdomen was inspected and no visible peristalsis could be seen. His abdomen was tender to palpation in the upper quadrant and epigastrum, without rebound tenderness and muscle tension. No masses were detected in the abdomen. Murphy's sign was negative. Neither enlargement of liver, spleen and kidneys beneath the rib was found, nor edema of lower limbs. Hepatorenal area was tender to palpation without rebound tenderness. Shifting dullness was positive, bowl sound was normal, without vessel murmur. There was no specialness in anorectal genitalia.

\subsection{Auxiliary examination}

Blood biochemical test: WBC $5.66 \times 10^{9} / L$, HGB $161 \mathrm{~g} / \mathrm{L}$, PLT $246 \times 10^{9} / L$. Blood gas analysis: $\mathrm{pH} 7.392, \mathrm{PCO}_{2}$ $39.1 \mathrm{mmHg}, \mathrm{PO}_{2} 77.4 \mathrm{mmHg}, \mathrm{BE}-1.4 \mathrm{mmol} / \mathrm{L}, \mathrm{HCO}_{3}^{-}$ $23.1 \mathrm{mmol} / \mathrm{L}$. Four infection index: HBsAg negative, AntiHCV negative, Anti-HIV negative, Anti-TP negative. Blood

\footnotetext{
*Correspondence: Shipeng Song; E-mail: songsp2009@126.com; Address: The Third Affiliated Hospital of Inner Mongolia Medical University, Hohhot, China.
} 
biochemical test: $\mathrm{K}^{+} 4.1 \mathrm{mmol} / \mathrm{L}, \mathrm{Na}^{+} 141 \mathrm{mmol} / \mathrm{L} ; \mathrm{Cl}^{-}$ $106 \mathrm{mmol} / \mathrm{L}$, GLU $5.32 \mathrm{mmol} / \mathrm{L}, \mathrm{Ca}^{2+} 2.23 \mathrm{mmol} / \mathrm{L}$. Liver function test: ALT $25 \mathrm{U} / \mathrm{L}$, AST $18 \mathrm{U} / \mathrm{L}$, TBIL $12 \mu \mathrm{mol} / \mathrm{L}$, DBIL $3.1 \mu \mathrm{mol} / \mathrm{L}$, ALB $42.4 \mathrm{~g} / \mathrm{L}$, TP $64.9 \mathrm{~g} / \mathrm{L}$. Four blood coagulation indexes: PT $11.7 \mathrm{~s}$, PTA $92.7 \%$, APTT $28.4 \mathrm{~s}$, TT 17.6 s, FBG 2.832 g/L. Stool routine test: fecal occult negative. Abdominal ultrasound: fatty liver, rough gallbladder wall. Chest X-ray: no abnormalities in the lungs, heart and diaphragm.

\subsection{Primary diagnosis}

Duodenal tumor of unknown origin: duodenal leiomyoma?

\subsection{Diagnosis and treatment}

The patient was eventually taken for surgery on November 25,2011 . We cut open the abdomen according to anatomical level, and found hepar and gall bladder normal. In the descending portion of duodenum and outside peritoneum, separation in the rear was made. A small needle suture stretch was performed at 12, 3, 6, 9 o'clock position of duodenum. A soft mass, approximately $2 \mathrm{~cm} \times 1 \mathrm{~cm}$, with smooth surface, good mobility, without blood, was detected in medial wall after $3 \mathrm{~cm}$ longitudinal incision. Complete resection was immediately taken after basal clamp being forceps clipped. Rapid pathological examination confirmed the diagnosis of heterotopic pancreas. 5-0 slip line continuous suture for the incision, 5-0 Vicryl transverse continuous suture for all layers and seromuscular layer, continuous suture for duodenum were done. Subsequently, 1 drainage tube is placed and fixed under the liver, and abdomen was oversewn layer by layer. During the postoperative courses, he was treated with cefuroxime sodium, levofloxacin anti-inflammatory, glutathione for liver protection, fat emulsion, amino acid, vitamin nutrition for nutritional therapy. Abdominal incision pain gradually subsided, abdominal drainage of $70 \mathrm{ml}$ pale bloody fluid, and then gradually reduced on postoperative day 1 . After a week, exsufflation and defecation returned to normal condition. He had liquids food when the nasogastric tube was pulled out. Blood biochemical test and biochemical examinations were normal, so that antibiotics were ceased then. No blood was remarkably found from abdominal cavity drainage tube on postoperative day 12 . There was no effusion abdominal and pelvic cavity during abdominal B-mode ultrasound examination. The drainage tube was pulled out then. Two weeks after the surgery, he was able to have semi liquid diet. Nutritive therapy, therefore, was quitted. The patient was discharged from the hospital on postoperative day 30 . He had normal appetite, defecation, and gained $5 \mathrm{~kg}$ weight during the follow-up visit. Postoperative pathology showed duodenal heterotopic pancreas so that diagnosis of duodenal heterotopic pancreas was confirmed.

\section{Discussion}

\subsection{Dr. Rui Liu}

Dr. Rui Liu is the Chief Physician of General Surgery Department at the Third Affiliated Hospital of Inner Mongolia Medical University, specializing in hepatobiliary surgery and breast disease.

Heterotopic pancreas (HP), also known as oberrant pancreas or accessory pancreas, is a congenital anomaly defined as pancreatic tissue occurring outside its normal anatomical location, lacking both anatomic and vascular connections. ${ }^{[1]} \mathrm{It}$ may occur at a variety of sites in the gastrointestinal tract, producing pancreatic enzymes and pancreatic juice. Heterotopic pancreas tissue is usually silent but can undergo complications that occur in normal pancreatic tissue such as acute or chronic pancreatitis, cyst or cancer. Heterotopic pancreas was first found by Schultz in an ileal diverticulum in 1727. ${ }^{[2]}$ Klob proved the pathological existence of the disease in 1859.The first case report of HP in China was made by Xueqiao Wang in 1957. HP occasionally presents symptomatically, and the manifestations vary depending on the location of the lesion. It is easy to be misdiagnosed during clinical practice. It can occur at any age, ranging from the newborn baby to 88 years old individuals, with an average age of 40.3 years old, and the ratio of male to female is $2: 1$. Heterotopic pancreas is mostly found in gastric antrum, duodenum, jejunum and Meckel diverticulum. Unusual localizations are the colon, spleen, liver biliary tract mesentery and lymph nodes. ${ }^{[3]}$ The duodenum is the most common site of heterotopic pancreas, especially the periampullary region. ${ }^{[4,5]}$ The statistical analysis of the results by Chen Wuxing, ${ }^{[6]}$ based on domestic related literature, generally consistent with the foreign reports, demonstrating that almost half of the cases of heterotopic pancreas were in stomach and duodenum.

The pathogenesis of heterotopic pancreatic tissue is unclear. The majority view believes that it is due to abnormal development of the embyro. Several theories have been proposed to explain the pathogenesis and occurrence of pancreatic heterotopia. ${ }^{[7]}$

(1) Aberrant primordium theory. Partial pancreatic primordia get non inflammatory adhesion with adjacent gastric, intestinal wall and mesentery during embryonic development. The aberrant primordia gradually separate from primary pancreatic primordium. Migration of this embryonic remnant along with the development of the gastrointestinal tract gives rise to the heterotopic pancreas in neighboring stomach, intestinal wall and mesentery.

(2) Embryo reversion theory. Heterotopic pancreas can occur in lung, mediastinum or other rare distant sites. Therefore, it is included that human reasoning is repeating phylogenetic history in the process of embryonic development. 
(3) Cell cultivation theory. The cells that were dropped from the dorsal pancreatic rudiment is often left to develop in a site away from the usual location of the body and tail of the pancreas, therefore, heterotopic pancreas arose in different organs.

(4) Incomplete atrophy theory. Minority primordia are pairs of left and right, with the left part shrinking gradually in the growth process. No-atrophy or incomplete atrophy triggers the formation of heterotopic pancreatic.

(5) Mesoderm conversion theory. Early primitive cells in other parts of the endoderm transformed into pancreatic cells during the development process, and grow into heterotopic pancreas. Heterotopic pancreas in our case was within duodenum, showing no specific clinical symptoms. The root reason for our case is inclined to aberrant primordium theory.

\subsection{Dr. Shengbin Zhang}

Dr. Shengbin Zhang is the Chief Physician of General Surgery Department at the Third Affiliated Hospital of Inner Mongolia Medical University, specializing in hepatobiliary surgery and organ transplantation.

Heterotopic pancreas (HP) is typically an asymptomatic congenital malformation, and its preoperative diagnosis is difficult so that it is easy to be unrecognized and missed diagnosed. ${ }^{[8]}$ Most often, HP is found in the stomach, duodenum and jejunum, ${ }^{[9]}$ but it may also be found anywhere in the digestive tract. It is generally located in the submucosal layer, muscularis layer and serous layer. Usually, it is a silent anomaly but it may become clinically evident when complicated with inflammation. ${ }^{[10]}$

In 1989, Armstrong et al. reported that over $50 \%$ of the clinical cases of heterotopic pancreas were without any symptoms, known as latent type among some scholars. Usually, the manifestations are concealed by associated lesions, but some are clinically significant. ${ }^{[11]}$ Symptoms depending upon the anatomical location, are also related to the size of the lesion and the adjacent organs. ${ }^{[11]}$ Also, they are classified into two types. (1) Pathological changes of heterotopic pancreas. Generally, heterotopic pancreas lesions are similar to any disease caused by normal pancreas, such as acute and chronic pancreatitis, pancreatic cyst, adenoma, adenocarcinoma, ${ }^{[12,13]}$ and clinically manifested as acute and chronic abdominal pain, tumor, digestive tract multiple ulcer (Zollinger-Ellison syndrome), Whipple's triad syndrome (episodes of hypoglycemia, blood glucose below L $2.8 \mathrm{mmol} / \mathrm{L}$, oral administration or intravenous injection of glucose could relieve the symptoms). (2) In rare cases, heterotopic pancreas may be detected by the mass in abdominal wall, spermatic cord and other parts. Postoperative pathology could confirm the diagnosis of the mass. ${ }^{[14]}$

In conclusion, the possibility of heterotopic pancreas is ac- ceptable given the symptoms of gastrointestinal ulcers, hypoglycemia and hyperinsulinemia are presented. Upper gastrointestinal heterotopic pancreas may appear non-specific gastrointestinal symptoms, similar to many symptoms associated with gastrointestinal ulcer, such as abdominal pain, pantothenic acid, belching, nausea, vomiting, hematemesis, melena, appetite and weight loss, complicated with digestive tract inflammation, ulcers, bleeding and obstruction, even canceration. While, heterotopic pancreas in hepatobiliary system or Vater's ampulla of duodenum may present as jaundice and fever. ${ }^{[12]}$ Despite the development of modern diagnostic modalities, its preoperative diagnosis remains challenging. Endoscopic ultrasonography, however, serves as a useful tool that aid in its identification with other diseases. ${ }^{[15]}$ In addition, heterotopic pancreas is also highly indicated in patients with submucousal bulging, intact mucosa, lesions of the central depression, tube structure, and secretions. ${ }^{[16]}$ Biopsy should be extended to submucosa and muscular layer several times. Namely, the golden standard for the final diagnosis is pathological diagnosis. Clinical symptoms of the patient are nonspecific, including: (1) abdominal pain and discomfort, (2) nausea, pantothenic acid, heartburn, black stool, weight loss, (3) duodenal endoscopic ultrasonography showed duodenal smooth muscle layer mass. Eventually, the diagnosis was established according to pathological examinations.

\subsection{Dr. Tianyong Zhao}

Dr. Tianyong Zhao is Associate Chief Physician of General Surgery Department at the Third Affiliated Hospital of Inner Mongolia Medical University, specializing in hepatobiliary surgery.

The manifestations vary depending on the location of the lesion:

\subsubsection{Ulcer}

symptom of peptic ulcer that caused by heterotopic pancreas in the stomach and duodenum or proximal jejunum area resembles those that caused by normal gastric and duodenal ulcer, such as regular upper abdominal burning or starvation pain, or upper abdominal pain that are much associated with starvation; varying degrees of acid reflux, belching. In addition, heterotopic pancreas occasionally leads to ulcer of mucosal ischemic necrosis due to local compression, exocrine pancreatic juice near the pylorus that effect gastric emptying function. Gastrointestinal hemorrhage, pyloric obstruction or perforation may occur with the appearance of corresponding symptoms and signs in clinic under joint effect of this kind of ulcer and heterotopic pancreas.

\subsubsection{Hemorrhage}

Chronic hemorrhage caused by ectopic pancreas is mainly presented as long-term melena and anemia. But it occasionally causes acute, massive hemorrhage, clinical vomiting, 
and even hemorrhagic shock.

\subsubsection{Obstruction}

Regardless of the volume that heterotopic pancreas occupy, the symptoms caused by heterotopic pancreas, such as peripheral inflammation, edema, ulcer and cicatricial tissue, contribute to luminal stenosis and obstruction. Pyloric obstruction in the vicinity of pylorus appears as abdominal distension, vomiting, gastric-pattern peristalsis and succusion splash. They can result in intestinal obstruction or intussusception in the small intestine, clinically manifested as abdominal distention, nausea, vomiting, anus exhaust and defecate or abdominal mass and bloody stool. The bile duct obstruction may occur because of heterotopic pancreas in the bile duct system, characterized by chills, ardent fever, right abdomen colic and jaundice.

\subsubsection{Diverticulosis}

Heterotopic pancreas can occur in the digestive tract of the diverticulum. It may also stir up the formation of digestive diverticulum. Further studies on their relationships remain to be done. Generally heterotopic pancreas may cause diverticular inflammation, bleeding, clinically appearing as chronic abdominal pain and melena similar to the clinical manifestations of gastritis, gastric ulcer and cholecystitis.

\subsection{Dr. Jin Zhao}

Dr. Jin Zhao is the Associate Chief Physician of General Surgery Department at the Third Affiliated Hospital of Inner Mongolia Medical University, specializing in gastrointestinal diseases.

\subsubsection{X-ray angiography}

Upper gastroenterography is advisable when heterotopic pancreas is suspected in the upper gastrointestinal tract. The classic radiological features of heterotopic pancreas are well-delineated submucosal filling defect with a characteristic central indentation, known as umbilication or dimple. The narrow dense shadow that continued with this barium plaque was called "ductal sign". Prepyloric filling defect, umbilical sign after pressure, and a tangent duct sign are highly suggestive of heterotopic pancreas in the literatures. ${ }^{[17]} \mathrm{X}$-ray gastrointestinal barium examination of heterotopic pancreas is often misdiagnosed as gastric, duodenal ulcer, gastric polyps and gastrointestinal tumors. The accuracy in the reported X-ray barium meal examination is $5.5 \%-71.4 \%$.

\subsubsection{Multislice CT}

Due to the special shape and limited anatomy of the digestive tract, smaller heterotopic pancreas is often unrecognized prior to the appearance of its complications. CT enhancement scan, however, is helpful in detecting the lesions of larger heterotopic pancreas or its complications. Round or oval masses with clear edge could be seen over CT examination. Heterotopic pancreatic tissue can enhance to the same degree as normal pancreas, showing homogeneous or inhomogeneous enhancement over enhaned scan. The majority of the cases are presented as complete duplicature with obvious enhancement in the surface. Gastric heterotopic pancreas could be widely connected with gastric wall, ${ }^{[18]}$ solitarily manifested as thickness of gastric wall, similar to pathological changes of liomyoma. The diagnosis of heterotopic pancreas was established over the appearance of "ductal sign". [19]

\subsubsection{CT}

CT virtual endoscopy is a newly-developed method in recent years, showing great diagnosis value in heterotopic pancreas of larger volume. It can display the structure of internal lesions as well as its site and size, which provides more accurate information for the diagnosis of heterotopic pancreas.

\subsubsection{Endoscopy}

Endoscopy not only shows heterotopic pancreas and pathological changes of surrounding organs but also allows examination of amylase in effluent liquid. Its greatest advantage lies in the fact that endoscopy examination enables the definite diagnosis by pathological biopsy, even the endoscopic resection of the disease.

\subsubsection{Endiscopic Ultrasonography}

Currently, the application of endiscopic ultrasonography in the diagnosis of heterotopic pancreas has been widely reported. The manifestations of heterotopic pancreas in the endoscopic ultrasonography vary, and the echoes could be medium or mixed. It can occur at any layer or multilayer within gastric wall, but the majority of the cases refer to submucosal layer. Endiscopic ultrasonography examination of the lesions will be of greater value if gland structure could be revealed. The patient in our case did not receive gastroenterography or CT examination. Gastroscope visualized a hemispherical bulging in duodenum, about $0.6 \mathrm{~cm}$ $* 0.8 \mathrm{~cm}$ in volume, with smooth surface, good mobility, and same color with surrounding mucosa. Duodenal endoscopic ultrasound examination showed a round mass with marked intact intestinal wall structure, no complete capsule or no signs of infiltration. In addition, fourth layer presented homogeneous hypoechoic foci. The biopsy was not performed in case the occurrence of bleeding so that preoperative diagnosis was not established.

\subsection{Dr. Zhijian Zhang}

Dr. Zhijian Zhang is the Associate Chief Physician of Pathology Department at the Third Affiliated Hospital of In- 
ner Mongolia Medical University, specializing in systemic clinical pathological diagnosis.

The gross appearance of heterotopic pancreas is a firm, yellow, finely lobulated nodule, which is usually located in the submucosa but sometimes extends into the muscularis or serosa, ranging from $0.5 \mathrm{~cm}$ to $5.0 \mathrm{~cm}$. It will be accompanied by cystic change or canceration on condition that the diameter greater than $5 \mathrm{~cm}$. Furthermore, it is distributed in the digestive tract submucosa (50\%-60\%), and some even proliferating invasively into the muscular layer (20\%-30\%) or involving all the layers. Heterotopic pancreas was without capsule and could not be peeled. The anatomy of heterotopic pancreas in different parts demonstrates its similarity with normal pancreas, containing a mixture of lobules, acini and ducts, and even islet. But the number of islet varies, which is much associated with degree of differentiation and distortion. Heterotopic pancreas has been classified into three types by Hcinrich in 1909, according to its constitutional components. Namely, type I consists of typical pancreatic tissues with acini, ducts, and islet cells similar to those seen in normal pancreas. Type II is composed of pancreatic tissues with ducts and acini. Islet cells are absent. Type III is composed of pancreatic tissue with large numbers of ducts and few acini. The number of islets in type I vary, ranging from 2-3 to 70-80 at high power magnification $(400 \times)$. Morphologically, heterotopic pancreas is composed of normal pancreatic acinar and ductal structure. Its existence could range from the submucosal layer to the proper muscular layer, proliferating invasively from the muscular layer to the serosa without any clinical symptoms for a long time. The small intestinal glands are visualized to be wrapped with acinar cell with nest-like distribution on the nodule. The nerve around the acinar tissue and adipose tissue around the islets also could be found. In most cases, smooth muscle hyperplasia at different degrees could be seen in the interstitial tissue of heterotopic pancreas, especially in patients with pyloric obstruction, surrounding acinar and duct segmentation. A varying number of chronic inflammatory cell infiltration would be discovered in the interstitial stroma. Pancreatic tissue and fibrous tissue hyperplasia is presented in pancreatic tissue with obstructive symptoms, characterized with inflammatory cell infiltration. Heterotopic pancreatic cyst and malignant transformation are suspected when the diameter extends to $6 \mathrm{~cm}$. Rare cases of carcinoma arising from heterotopic pancreas were reported in malignant tissues. Patients with tumor changes are often not accompanied by lymph node invasion. ${ }^{[12]}$ The diagnosis of heterotopic pancreas, differentiating with other pancreatic tumors heavily relies on immunohistochemistry, ${ }^{[20]}$ a comprehensive analysis of ${ }^{[21]}$ morphology, immunohistochemical characteristics and genes of it, such as the overexpression of P53. ${ }^{[22]}$

Published by New Century Science Press

\subsection{Dr. Shipeng Song}

Dr. Shipeng Song is the Associate Chief Physician of General surgery Department at the Third Affiliated Hospital of Inner Mongolia Medical University, specializing in minimally invasive surgery, organ transplantation.

Heterotopic pancreas is a rare disease, mainly presenting in the digestive tract, causing ulcers, bleeding, obstruction, intussusception and even tumor. Clinically, undiagnosis and missed diagnosis often occur. In addition, heterotopic pancreas could potentially change into cystic degeneration, necrosis, hemorrhage, and heterotopic pancreatitis and cancer. $^{[23]}$ In that case, surgical management, especially minimally invasive surgery, is advisable whenever heterotopic pancreas is found with or without symptoms. ${ }^{[24]}$ Surgical options of the disease depend on the location of the lesion. Local excision is appropriate, only if the cancer lesions occur. Extended excision should not be performed in order to avoid increased surgical complications. During the surgery, a quick routine biopsy should be done to establish the diagnosis. Local excision is feasible for heterotopic pancreatitis in the stomach, duodenal bulb resection and Billroth II gastrojejunostomy for the gastric antrum and duodenal ectopic pancreas respectively. Local resection and subsequent bile duct plasty should be required in case that heterotopic pancreas is small in volume, and has clear boundaries with Vater's ampulla. If not, pancreaticoduodenectomy is highly recommended. As for the pathological changes of heterotopic pancreas in ileum and horizontal or ascending part of duodenum, partial intestine resection and anastomosis is needed. Preoperative endoscopic ultrasonography is of assistance in the initial selection of surgical methods. If heterotopic pancreas originates in the submucosa, without muscular or serosal layer involved under ultrasound endoscopes examination, endoscopic high-frequency electroexcision is indicated. Physiological saline treatment around the base of the lesions is feasible for comparative superficial bulging, and snare excision was subsequently performed after elevation of lesions. If heterotopic pancreas gets connected with the muscular layer or serosa, appropriate surgical resection should be performed. ${ }^{[25]}$ In our case, the patient underwent partial resection, recovered well and was discharged then without signs of pancreatic fistula, intestinal fistula and other complications.

\section{Conclusions}

In conclusion, heterotopic pancreas is a rare disease without typical manifestations so that preoperative diagnosis is challenging. It is very important for us to raise awareness of the disease, in order to facilitate the accurate diagnosis and treatment, prevention of complications. The possibility of heterotopic pancreas is acceptable if the following conditions are met: (1) Unexplained abdominal distension 
and pain (2) atypical peptic ulcer (3) Refractory ulcer and biliary diseases (4) Unexplained pyloric obstruction (5) intestinal obstruction (6) Unexplained obstructive jaundice (7) Abdominal mass (8) Unexplained gastrointestinal bleeding. Timely gastrointestinal barium X-ray and endoscopy exam-

\section{References}

[1] Kui Shen, Shouxian Zhong, Shengdao Zhang. Pancreatic surgery. Beijing: People's Medical Publishing House, 2000, 292.

[2] Beleta E, Peghin M, Guarner L. Cystic dystrophy of the pancreas in patient with only one previous cyst [J]. Rev Esp Enferm Dig, 2011, 103: 95-97.

[3] Yaseshima KS. An antopsy case of pancreatic and ectopic nesidioblastosis [J]. Pathol Int, 2001, 51(5): 376-379.

[4] Chen CH, Yang CC, Yeh YH, et al . Ectopic pamnreas located in the major duodenal papilla: Case report and review [J]. Gast roint est Endosc, 2001, 53(1) : 121-123.

[5] A Tanemura, T Yano, H Tamaki, et al . Ectopic pancreas in the minor duodenal papilla presenting as upper-GI bleeding [J]. Gastrointest Endosc, 2005, 62(2): 321-326.

[6] Wuxing Chen, Guangping Chen. Anatomy and clinical study of heterotopic pancreas [J]. Journal of Anatomy, 2006, 29(4): 516-518.

[7] Hebrok M, Kim SK, St Jacques B, et al. Regulation of pancreas development by hedgehog signaling. Development, 2000, 127(22): 4905-4907.

[8] Hongbo Wang, Mingze Ma, Chongmei Yang, et al. Diagnosis and treatment of 60 cases of heterotopic pancreas in gastrointestinal tract [J]. Chinese Journal of Pancreatology, 2010, 10(4): 238-240.

[9] Yuan Z, Chen J, Zheng Q, et al. Heterotopic pancreas in the gastrointestinal tract $[\mathrm{J}]$. World J of Gastroenterol, 2009 Aug 7; 15(29): 3701-3.

[10] Taranu T, Vintila D, Neacsu N, et al. Heterotopic accessory pancreas in surgical pathology: review of 23 years experience $[\mathrm{J}]$. Chir $(\mathrm{Bu}-$ cur), 2010, 105(3): 347-353.

[11] Mat suki M, Gouda Y,Ando T, et al. Adenocarcinoma arising from aberrant pancreas in the stomach. Gastroent erology, 2005, 11(1): 652-656.

[12] Jeong HY, Yang HW, Seo SW. Adenocarcinoma arising from an ectopic pancreas in the stomach. Endoscopy, 2002, 34(12): 10141018. ination are highly recommended.

\section{Conflicts of Interest Disclosure}

The authors have no conflict of interest related to this article.
[13] Y YH, Guo WH, Yu SY. Mesenteric heterotopic functional islet cell tumor: A case report and literature review. Chinese medical journal of misdiagnosis, 2005, 10(1): 13-15.

[14] Hongfu Sun. Ectopic pancreas in the inner cord: a case report. Qinghai Medical Journal. 1999, 29(12): 64.

[15] Shen Y, Wang BM, Fang WL, et al. The value of endoscopic ultrasonography in the diagnosis and treatment of ectopic pancreas in upper digestive tract [J]. Chinese Journal of Digestive Endoscopy, 2010, 27(6): 287-290

[16] Payeras G, Castellon C, De Jaime J, et al. Heterotopic pancreas: a difficult diagnosis[J]. Endoscopy, 2010, 42 Suppl 2: E121. Epub 2010 Mar 19.

[17] Want JF, Wang YK, Mo YW. Diagnosis of ectopic pancreas in 7 cases of abdominal operation [J]. Chinese Journal of Misdiagnostics, 2005, 5(16): 3106.

[18] Lu LJ, Li P, Lei XY. One case of heterotopic pancreas in the stomach [J]. Chinese Journal of Clinical Medical Imaging, 2008, 19(7): 497.

[19] Wu GY, Tian ZX, Zhang ZP, et al. CT manifestations of ectopic pancreas in the stomach [J]. Chinese Journal of Radiology, 2007, 41(9): 938-940.

[20] Ornoczky T, Kalman E, Jakso P, et al. Solid and papillary epithelialneoplasm arising in heterotopic pancreatic tissue of the mesocolon [J]. J Clin Pathol, 2001, 54(3): 241-245.

[21] Osanai M, Miyokawa N, Tamaki T, et al. Adenocarcinoma arising in gastric heterotopic pancreas:Clinicopathological and immunohistochemical study with genetic analysis of acase [J]. Pathol Int, 2001, 51(7): 549-554.

[22] Nisar PJ, Zaitoun AM, Lobo DN, et al. Heterotopic pancreas in the spleen: malignant degeneration to mucinous cyst adenocarcinoma [J]. Eur J Gastroenterol Hepatol, 2002, 14(7): 793-796.

[23] Rimal D, Thapa SR, Munasinghe N, et al. Symptomatic gastric heterotopic pancreas:Clinical presentation and reviewof the literature [J]. Int J Surg, 2008 Dec; 6(6): e52-4. Epub 2007 Feb 12.

[24] Margolin DJ. Endoscopy-assisted laparoscopic resection of gastric heterotopic pancreas [J]. Am Surg, 2008 Sep; 74(9): 829-31.

[25] Furukawa T, Watanabe M, Ozawa S, et al. Development of endoscopic surgery for the minimally invasive treatment of digestive and other diseases [J]. Keio J Med, 2001, 50(3): 167-174. 